\title{
Estimación de la Incidencia de Enfermedades Ocupacionales en Colombia, 1985-2000
}

\begin{abstract}
Alvaro Javier Idrovo
Médico, Especialista en Higiene y Salud Ocupacional, M. Sc. Salud Pública, MSc Salud Ambiental, Candidato a Doctor en Epidemiología. Instituto Nacional de Salud Pública. Cuernavaca, México. E-mail: idrovoaj@hotmail.com.
\end{abstract}

\section{RESUMEN}

Objetivos En Colombia no se conoce la ocurrencia de enfermedades relacionadas con el trabajo. El objetivo de este estudio es estimar la incidencia de la enfermedad ocupacional en Colombia en el periodo comprendido entre 1985 y 2000.

Metodología Con base en la metodología de Leigh y colaboradores, y con las estimaciones poblacionales del Departamento Administrativo Nacional de Estadística (DANE) se estimó indirectamente la incidencia anual.

Resultados Los resultados mostraron un incremento en la incidencia de enfermedades ocupacionales desde 68063 casos en 1985 a 101645 en 2000.

Discusión Se consideran las pérdidas económicas que el subregistro de enfermedades ocupacionales ocasiona al sistema de seguridad social en salud.

Palabras Claves: Salud ocupacional, epidemiología, Colombia (fuente: DeCS, BIREME).

\section{ABSTRACT \\ Estimation of the incidence of occupational diseases in Colombia, 1985-2000}

Objectives The occurrence of labor related diseases in Colombia is unknown. The objective of this study was to estimate the incidence of occupational disease in Colombia in the period 1985-2000.

Methods The annual incidence was estimated with the methodology recommended by Leigh et al. based upon the population estimates of the National Administrative Statistics Department (DANE).

Results The results showed an increase in the incidence of occupational diseases from 68063 cases in 1985 to 101645 in 2000. 
Discussion The economic losses caused to the social security health system by underreporting of occupational diseases are estimated.

Key Words: Occupational disease, occupational health, epidemiology, Colombia (source: $\mathrm{MeSH}, \mathrm{NLM}$ ).

L a íntima relación entre la salud y el trabajo es conocida desde tiempos inmemoriales. Muchas exposiciones a agentes y condiciones presentes en el ambiente laboral son reconocidas como factores de riesgo implicados en la patogénesis de muchas enfermedades (1), pese a que su ocurrencia es desconocida en casi todos los países. Las enfermedades ocupacionales son enfermedades, por lo menos en teoría, prevenibles que ocasionan grandes pérdidas económicas por la disminución de la productividad y los costos requeridos para su atención (2). Por tal razón su cuantificación es un paso básico para definir las políticas que tengan por objetivo reducir su ocurrencia.

En el contexto de la seguridad social colombiana las enfermedades ocupacionales también tienen un impacto importante. Cuando una enfermedad ocupacional es diagnosticada a un trabajador que ha sido afiliado al sistema de riesgos profesionales durante el tiempo correspondiente al periodo de latencia, los costos de diagnóstico, tratamiento y rehabilitación deben ser asumidos por este sistema, lo cual significa un reembolso de los costos que hasta ese momento pueden estar teniendo las empresas promotoras de salud o las administradoras del régimen subsidiado.

Infortunadamente la falta de profesionales clínicos capacitados en el diagnóstico de enfermedades ocupacionales, incluyendo a los especialistas en salud ocupacional (3), acompañada de una normatividad (Decreto 1295 de 1994, Decreto 1832 de 1994, Decreto 917 de 1999 y Resolución 2569 de 1999, por solo citar algunas) que dificulta la calificación de una enfermedad como laboral al considerar mecanismos unicausales, lejanos a la multicausalidad propia de las enfermedades humanas (4), tienen como resultado un subregistro exagerado de la ocurrencia de este tipo de enfermedades.

Ante esta realidad, el objetivo propuesto para el presente trabajo fue el de estimar la incidencia de algunas enfermedades ocupacionales en Colombia de 1985 al año 2000, como un primer paso en la discusión del impacto económico que pueden tener sobre el sistema de seguridad social en salud. De esta manera se busca acabar con el mito de la no ocurrencia de enfermedades relacionadas con el trabajo en el país, y seguir con la tendencia internacional de realizar estimaciones de este tipo (5-8) para definir políticas de salud. 


\section{MATERIALES Y MÉTODOS}

La incidencia nacional de enfermedades ocupacionales para cada uno los años entre 1985 y 2000 fue estimada con el método de aproximación indirecto descrito por Leigh y colaboradores (9). En resumen, se partió del doble de la incidencia de casos de enfermedad ocupacional reportada en Finlandia durante 1993, que muestra la incidencia específica por edad, sexo y enfermedad (10) tal como se indica en la Tabla 1. La incidencia por categorías de edad y sexo no indican que en estas edades se presenten las enfermedades, sino que es un artificio para facilitar los cálculos.

Tabla 1. Incidencia de enfermedades ocupacionales asumida en los cálculos (tasas por millón de habitantes)

\begin{tabular}{|c|c|c|c|c|c|}
\hline & \multirow[b]{2}{*}{ Enfermedad } & \multicolumn{4}{|c|}{ Edad (años) } \\
\hline & & $0-14$ & $15-44$ & $45-60$ & $60+$ \\
\hline \multirow{9}{*}{$\begin{array}{l}\mathscr{d} \\
\text { 을 } \\
\text { 우 }\end{array}$} & Neumoconiosis & 0 & 172 & 492 & 590 \\
\hline & Enfermedad respiratoria crónica & 8 & 344 & 3686 & 3550 \\
\hline & Alteraciones musculoesqueléticas & 62 & 1488 & 982 & 118 \\
\hline & Cáncer & 0 & 9 & 294 & 590 \\
\hline & Trastornos neuropsiquiátricos & 0 & 86 & 246 & 60 \\
\hline & Intoxicaciones por plaguicidas & 8 & 9 & 24 & 12 \\
\hline & Otras intoxicaciones & 8 & 42 & 98 & 30 \\
\hline & Dermatosis & 20 & 530 & 546 & 24 \\
\hline & Hipoacusia inducida por ruido & 0 & 354 & 3778 & 1112 \\
\hline \multirow{9}{*}{$\frac{\mathscr{d}}{\frac{d}{2}}$} & Neumoconiosis & 0 & 0 & 144 & 108 \\
\hline & Enfermedad respiratoria crónica & 4 & 450 & 960 & 108 \\
\hline & Alteraciones musculoesqueléticas & 128 & 1080 & 960 & 36 \\
\hline & Cáncer & 0 & 0 & 24 & 18 \\
\hline & Trastornos neuropsiquiátricos & 0 & 90 & 240 & 36 \\
\hline & Intoxicaciones por plaguicidas & 9 & 9 & 24 & 7 \\
\hline & Otras intoxicaciones & 9 & 18 & 48 & 18 \\
\hline & Dermatosis & 48 & 830 & 1118 & 26 \\
\hline & Hipoacusia inducida por ruido & 0 & 16 & 254 & 62 \\
\hline
\end{tabular}

La elección de los registros de Finlandia como patrón de comparación se debe a que se considera como el mejor en la actualidad, y el multiplicar por dos su ocurrencia es un factor de corrección debido a la menor capacidad de diagnóstico de enfermedades ocupacionales y al menor grado de desarrollo de la salud ocupacional en Colombia. Solo se incluyeron las siguientes enfermedades: neumoconiosis específicas, enfermedades crónicas respiratorias, alteraciones musculoesqueléticas, cáncer, trastornos neuropsiquiátricos, intoxicaciones por plaguicidas, otras intoxicaciones, dermatosis e hipoacusia inducida por el ruido, de acuerdo a las categorías de Murray y López (11), que pueden servir como indicadores de la ocurrencia global de las enfermedades ocupacionales. Las poblaciones nacionales anuales por grupos de edad y sexo fueron obtenidas de las proyecciones del Departamento Administra- 
tivo Nacional de Estadística-DANE (12). Solo se incluyeron los años entre 1985 y 2000 debido a que la metodología utilizada solo tiene en cuenta cambios en la composición demográfica, y podría errar al no incluir elementos como las modificaciones en las exposiciones ocupacionales principalmente debidas al desarrollo de los diferentes sectores económicos, y otros procesos sociales que tienen repercusión en la distribución del empleo.

\section{RESULTADOS}

La incidencia estimada de enfermedades ocupacionales en Colombia entre 1985 y 2000 va en aumento, empezando con cifras cercanas a los 68063 casos y llegando a los 101645 en el 2000. En la Figura 1 se pueden apreciar las cifras estimadas para cada uno de los 16 años estudiados, en total y por sexo.

Figura 1. Incidencia anual de enfermedades ocupacionales estimada para Colombia, 1985-2000

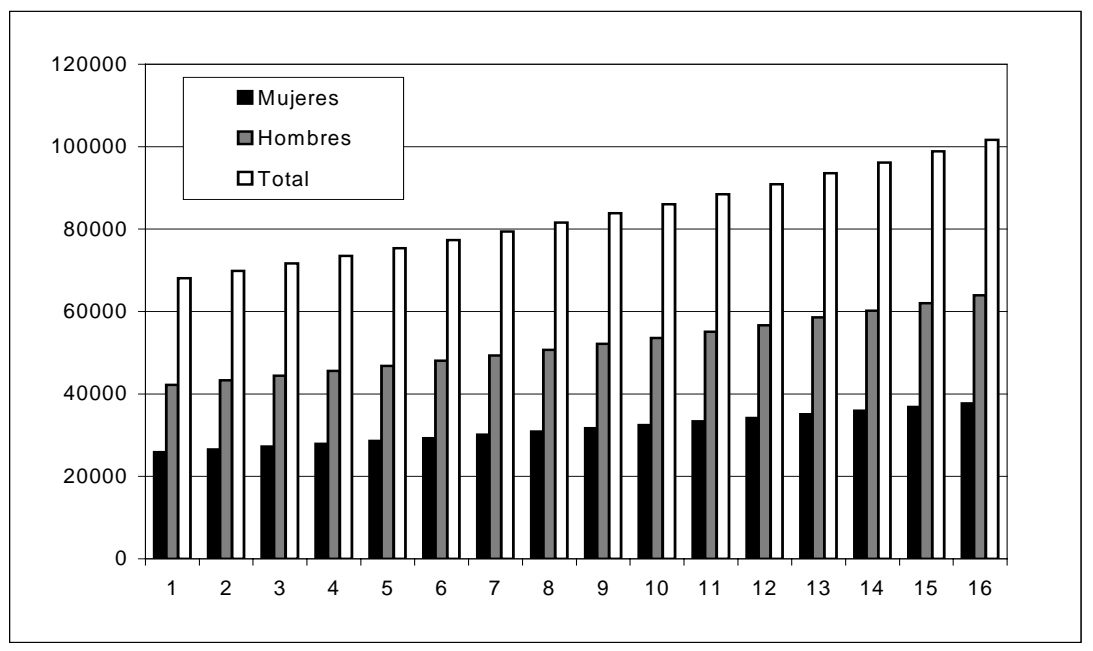

Las incidencias estimadas para cada una de las enfermedades incluidas en el análisis entre 1985 y 2000 se pueden observar en la Tabla 2. Como se puede apreciar, se espera que con excepción de las dermatosis la mayoría de las enfermedades afecte principalmente a los hombres. Las enfermedades más frecuentes serían las hipoacusias inducidas por ruido, las enfermedades respiratorias crónicas y las alteraciones musculoesqueléticas. 
Tabla 2. Estimación de la incidencia de enfermedades ocupacionales en Colombia, $1985-2000$

\begin{tabular}{|c|c|c|c|c|c|c|c|c|c|}
\hline \multirow[b]{2}{*}{ Año } & \multicolumn{9}{|c|}{ Enfermedad Ocupacional } \\
\hline & 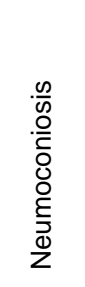 & 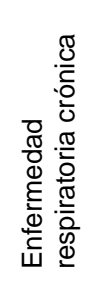 & 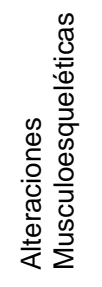 & 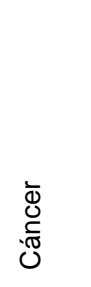 & 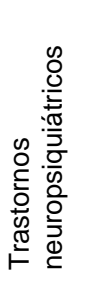 & 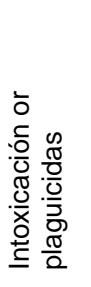 & 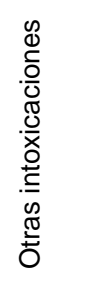 & 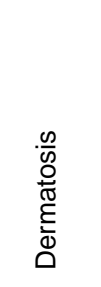 & 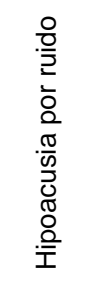 \\
\hline 1985 & 2753 & 15396 & 23667 & 1011 & 2074 & 316 & 791 & 13001 & 9054 \\
\hline 1986 & 2827 & 15797 & 24288 & 1039 & 2129 & 327 & 810 & 13345 & 9292 \\
\hline 1987 & 2902 & 16229 & 24903 & 1067 & 2185 & 330 & 830 & 13688 & 9541 \\
\hline 1988 & 2978 & 16659 & 25510 & 1096 & 2242 & 337 & 850 & 14028 & 9800 \\
\hline 1989 & 3055 & 17110 & 26111 & 1127 & 2300 & 344 & 870 & 14370 & 10076 \\
\hline 1990 & 3140 & 17605 & 26736 & 1161 & 2362 & 351 & 892 & 14732 & 10385 \\
\hline 1991 & 3227 & 18122 & 27368 & 1197 & 2428 & 360 & 914 & 15101 & 10712 \\
\hline 1992 & 3319 & 18671 & 28019 & 1235 & 2497 & 368 & 937 & 15485 & 11066 \\
\hline 1993 & 3414 & 19243 & 28666 & 1274 & 2567 & 376 & 961 & 15870 & 11440 \\
\hline 1994 & 3511 & 19836 & 29307 & 1315 & 2639 & 385 & 986 & 16257 & 11830 \\
\hline 1995 & 3614 & 20470 & 29949 & 1362 & 2715 & 393 & 1011 & 16652 & 12250 \\
\hline 1996 & 3722 & 21130 & 30618 & 1408 & 2795 & 403 & 1037 & 17061 & 12697 \\
\hline 1997 & 3840 & 21849 & 31322 & 1460 & 2880 & 413 & 1066 & 17580 & 13193 \\
\hline 1998 & 3961 & 22588 & 32010 & 1514 & 2966 & 423 & 1095 & 17913 & 13708 \\
\hline 1999 & 4084 & 23346 & 32696 & 1570 & 3053 & 433 & 1123 & 18339 & 14233 \\
\hline 2000 & 4213 & 24134 & 33385 & 1630 & 3143 & 442 & 1153 & 18771 & 14775 \\
\hline
\end{tabular}

\section{DISCUSIÓN}

Antes de cualquier comentario sobre las estimaciones hechas en este trabajo, es necesario tener en cuenta algunas consideraciones metodológicas. Las cifras presentadas corresponden únicamente a valores aproximados obtenidos mediante una metodología previamente usada para estos fines, y en ningún momento deben ser asumidos como las cifras "reales" de la ocurrencia de enfermedad ocupacional en Colombia. El factor de corrección hecho al registro finlandés puede ser objeto de muchas críticas. Sin embargo, ya ha sido utilizado para estimar la ocurrencia de enfermedades ocupacionales en todos los países que no tienen economías de mercado o socialistas establecidas. El resultado en los casos en que se ha podido comparar con los registros existentes u otras estimaciones, es que el método aquí utilizado es mucho más conservador y puede incluso estar mostrando un subregistro (9). Por tal razón, es razonable pensar que los hallazgos aquí descritos se pueden considerar como una subestimación de la ocurrencia real de enfermedades ocupacionales en Colombia. 
La forma de estimación usada, al ser uniforme para todos los años tiende a linealizar los hallazgos. Si bien esta es una fuerte debilidad del estudio, es una primera aproximación válida a la problemática en cuestión. Los pocos años alrededor de 1993 (año del que se obtuvo la ocurrencia en Finlandia) incluidos para el análisis corresponden a una consideración metodológica de cautela, debido a que el estimar la ocurrencia durante más años puede tener como resultado valores errados, ya que no consideraría los posibles cambios en la economía y en la población trabajadora. Posteriores estimaciones deberán tener en cuenta el desarrollo de los diferentes sectores de la economía, el desempleo, el sector informal de la economía y las características de las exposiciones laborales, entre otras variables.

Teniendo estas consideraciones en mente, los resultados presentados en este trabajo deben someterse a la pregunta: ¿realmente son válidos estos estimadores para Colombia? Si existieran unos registros que permitieran hacer una comparación directa podría explorarse la correlación existente con los datos aquí presentados. Sin embargo la falta de registros hace imposible esta tarea, por lo que estas cifras pueden considerarse como las mejores disponibles en el momento. En la actualidad los registros oficiales existentes solo son capaces de mostrar la punta más alta del iceberg de ocurrencia de la enfermedad ocupacional, tal como ha ocurrido en otros países (13).

Las implicaciones que tienen los hallazgos aquí presentados tienen enorme importancia en el campo de la salud pública y de la seguridad social. Desde que se encuentra vigente la Ley 100 de 1993 la seguridad social en Colombia tuvo un cambio radical al crear dos subsistemas para la atención de los eventos adversos sobre la salud: uno dedicado a las enfermedades y lesiones asociadas con el trabajo, y otro a las enfermedades y lesiones cuyo origen se considera no ocupacional. Sin embargo, siempre se parte del supuesto de que una enfermedad es no ocupacional, y se requiere de una extensa serie de actividades que tienen por objetivo tratar de identificar la relación entre las exposiciones laborales y la enfermedad en cuestión. Este proceso consume bastante tiempo y dinero a la institución aseguradora, la empresa y al trabajador, debido a que en la mayoría de los casos no se cuenta con la información adecuada requerida de manera inmediata. En todo este largo proceso el más afectado será el trabajador, que deberá someterse a un sinnúmero de exámenes sin que, en algunos casos, tengan un claro beneficio para él.

Para tener una idea general de las perdidas económicas generadas por el sistema de riesgos profesionales al sistema de seguridad social en salud, en 
Estados Unidos el tratamiento y compensación del dolor lumbar ocupacional puede llegar a necesitar hasta 1600 millones de dólares por año (14). De manera similar, para 1985 en ese país se estimó que los costos debidos a dermatosis ocupacionales oscilaba entre 250 y 1250 millones de dólares (15). En estos estudios solo se cuantificaron los costos económicos y no se tienen en cuenta el sufrimiento y las dificultades que pueden ocasionarse en el trabajador, su familia y sus dependientes económicos (1).

En conclusión, las enfermedades ocupacionales tienen una ocurrencia importante en Colombia, cuyas cifras reales pueden estar cerca de los descritos en este trabajo. Si bien no se tiene un alto grado de precisión, los datos aquí presentados pueden dar una idea de la problemática de la morbilidad ocupacional en Colombia. Las estimaciones aquí mostradas pueden servir de punto de partida para los tomadores de decisiones (16), así como incentivo a los profesionales e investigadores del campo de la salud ocupacional para realizar estudios observacionales que tengan por objetivo evidenciar la asociación existente entre los factores de riesgo ocupacionales y las enfermedades que aquejan a la población colombiana, mediante la incorporación de técnicas y métodos innovadores, dejando de lado la ceguera que impera actualmente ante esta problemática.

Por ejemplo se pueden vigilar estrechamente los eventos centinela ocupacionales $(17,18)$ y determinar su relación con los factores de riesgo presentes en los ambientes laborales en el país, o cruzar los registros de mortalidad y egresos hospitalarios con los trabajadores de los afiliados en cada una de las administradoras de riesgos profesionales, siguiendo métodos similares a los descritos por Marsh (19). De igual manera, se pueden usar los métodos epidemiológicos desarrollados recientemente para determinar la contribución etiológica proporcional de los factores ocupacionales de riesgo (20), en casos específicos con posible relación con el trabajo.

La calidad de los profesionales de la salud ocupacional y del grado de justicia social de los subsistemas de la seguridad social podrán medirse en la mejoría de los registros de ocurrencia de los eventos relacionados con el trabajo. Deberán modificarse las acciones de los profesionales de la salud dedicados a la salud ocupacional, desde la visión clínica a una epidemiológica $(21,22)$ más contextualizada, con la que se pueda lograr una mejor salud de la población trabajadora • 


\section{REFERENCIAS}

1. Abrams HK. A short history of occupational health. J. Public Health Pol. 2001; 22:34-80.

2. Weil D. Valuing the economic consequences of work injury and illness: a comparison of methods and findings. Am. J. Ind. Med 2001; 40:418-437.

3. De la Hoz RE, Guerrero E, Espinosa MT, de Fex RL. Occupational and environmental medicine in Colombia. Int. Arch. Occup. Environ. Health 2000; 73: 145-149.

4. Smith KR, Corvalán CF, Kjellstrom T. How much global ill health is attributable to environmental factors? Epidemiology 1999; 10:573-584.

5. Kraut A. Estimates of the extent of morbidity and mortality due to occupational diseases in Canada. Am. J. Ind. Med 1994; 25:267-278.

6. Leigh JP, Cone JE, Harrison R. Cost of occupational injuries and illness in California. Prev. Med 2001; 32:393-406.

7. Leigh JP, Markowitz SB, Fahs MC, Shin C, Landrigan PJ. Occupational injury and illness: estimates of costs, mortality and morbidity. Arch. Intern. Med 1997; 157:1557-1568.

8. Takala J. Global estimates of fatal occupational accidents. Epidemiology 1999; 10:640-646.

9. Leigh J, Macaskill P, Kuosma E, Mandryk J. Global burden of disease and injury due to occupational factors. Epidemiology 1999; 10:626-631.

10. Finnish Institute of Occupational Health. Occupational diseases in Finland in 1993. Helsinki: Finnish Institute of Occupational Health; 1994.

11. Murray CJL, López AD (eds.). The global burden of disease Vol. 1. Cambridge: Harvard School of Public Health, 1994; p. 27-33.

12. Departamento Administrativo Nacional de Estadística. Estimaciones de la población colombiana. 1985-2000.

13. Berman DM. Death on the job. London: Monthly Review Press; 1978.

14. Snook SH, Fine LJ, Silverstein BA. Musculoskeletal disorders. In: Levy BS, Wegman DH (eds.). Occupational health. Recognizing and preventing work-related disease $2^{\text {nd }}$ ed. Boston: Little, Brown and Company; 1988. p 345-370.

15. Mathias CGT. The cost of occupational skin disease. Arch. Dermatol 1985; 121: 332-334.

16. Pruss A, Corvalán CF, Pastides H, de Hollander AEM. Methodologic considerations in estimating burden of disease from environmental risk factors at national and global levels. Int. J. Occup. Environ. Health 2001; 7:58-67.

17. Rutstein DD, Mullan RJ, Frazier TD, Halperin WE, Melius JM, Sestito JP. Sentinel health events (occupational): a basis for physician recognition and public health surveillance. Am. J. Public Health 1983; 73:1054-1061.

18. Mullan RJ, Murthy LI. Occupational sentinel health events: an up-dated list for physician recognition and public health surveillance. Am. J. Ind. Med 1991; 19:775-799. 
19. Marsh GM. A strategy for merging and analyzing work history data in industry wide occupational epidemiological studies. Am. Ind. Hyg. Assoc. J 1987; 48:414-419.

20. McElduff P, Attia J, Ewald B, Cockburn J, Heller R. Estimating the contribution of individual risk factors to disease in a persona with more than one risk factor. J. Clin. Epidemiol 2002; 55:588-592.

21. Idrovo AJ. Rol del médico en un programa empresarial de salud ocupacional. Rev. Fac. Med. UN Col 1999; 47:35-43.

22. Wegman DH. The potential impact of epidemiology on the prevention of occupational disease. Am. J. Public Health 1992; 82:944-954. 